\title{
Optical interferometric temperature sensors for intravascular blood flow measurements
}

Elizabeth Carr, Eleanor C. Mackle, Malcolm C. Finlay, Charles A. Mosse, Joanna M. Coote, et al.

Elizabeth Carr, Eleanor C. Mackle, Malcolm C. Finlay, Charles A. Mosse, Joanna M. Coote, loannis Papakonstantinou, Adrien E. Desjardins, "Optical interferometric temperature sensors for intravascular blood flow measurements," Proc. SPIE 11075, Novel Biophotonics Techniques and Applications V, 1107502 (22 July 2019); doi: 10.1117/12.2527056 


\title{
Optical Interferometric Temperature Sensors for Intravascular Blood Flow Measurements
}

\author{
Elizabeth Carr ${ }^{\mathrm{a}, \mathrm{b}}$, Eleanor C. Mackle ${ }^{\mathrm{a}, \mathrm{b}}$, Malcom C. Finlay ${ }^{\mathrm{b}, \mathrm{c}}$, Charles A. Mosse ${ }^{\mathrm{a}, \mathrm{b}}$, \\ Joanna M. Coote ${ }^{\mathrm{a}, \mathrm{b}}$, Ioannis Papakonstantinou ${ }^{\mathrm{d}}$, Adrien E. Desjardins ${ }^{\mathrm{a}, \mathrm{b}}$ \\ ${ }^{a}$ Department of Medical Physics and Bioengineering, University College London, London, UK; \\ ${ }^{b}$ Wellcome/EPSRC Centre for Interventional and Surgical Sciences, University College London, Charles Bell \\ House, London, UK; 'Barts Heart Centre, Barts Health NHS Trust, London, UK; ${ }^{\mathrm{d} D e p a r t m e n t ~ o f ~ E l e c t r o n i c ~}$ \\ and Electrical Engineering, University College London, London, UK
}

\begin{abstract}
Direct and continuous measurements of blood flow are of significant interest in many medical specialties. In cardiology, intravascular physiological measurements can be of critical importance to determine whether coronary stenting should be performed. Intravascular pressure is a physiological parameter that is frequently measured in clinical practice. An increasing body of evidence suggests that direct measurements of blood flow, as additional physiological parameters, could improve decision making.

In this study, we developed a novel fibre optic intravascular flow sensor, which enabled time-of-flight measurements by upstream thermal tagging of blood. This flow sensor comprised a temperature sensitive polymer dome at the distal end of a single mode optical fibre. The dome was continuously interrogated by low coherence interferometry to measure thermally-induced length changes with nanometre-scale resolution. Flow measurements were performed by delivering heat upstream from the sensor with a separate optical fibre, and monitoring the temperature downstream at the dome with a sample rate of $50 \mathrm{~Hz}$.

A fabricated flow sensor was characterized and tested within a benchtop phantom, which comprised vessels with lumen diameters that ranged from 2.5 to $5 \mathrm{~mm}$. Water was used as a blood mimicking fluid. For each vessel diameter, a pump provided constant volumetric flow at rates in the range of 5 to $200 \mathrm{ml} / \mathrm{min}$. This range was chosen to represent flow rates encountered in healthy human vessels. Laser light pulses with a wavelength of $1470 \mathrm{~nm}$ and durations of $0.4 \mathrm{~s}$ were used to perform upstream thermal tagging. These pulses resulted in downstream temperature profiles that varied with the volumetric flow rate.
\end{abstract}

Keywords: Flow, pressure, coronary artery disease, low coherence interferometry

\section{INTRODUCTION}

Coronary artery disease (CAD) is a leading cause of death and disability in the developed world ${ }^{1}$, and it is responsible for approximately one-third of deaths in individuals over the age of $35^{2}$. It can be caused by atherosclerosis ${ }^{3}$, where lipid-rich plaques can rupture and restrict blood flow. Stenting is an important treatment option, but it carries significant risks, including blood clots and re-stenosis ${ }^{4}$. It is therefore of paramount importance to optimise decisions about whether stenting is appropriate.

Intravascular physiological measurements can be valuable to inform percutaneous treatments of CAD. Pressure measurements are currently in widespread use for this purpose. However, pressure-based parameters such as Fractional Flow Reserve (FFR) only provide indirect measures of flow. Indeed, FFR is based upon the assumption that the pressureflow relationship during maximal vasodilation is linear ${ }^{5}$, but this assumption may not hold if there are other diseases present. In particular, microvascular dysfunction can have a significant impact on FFR measurements ${ }^{6,7}$, as it impairs coronary vasodilation. Obtaining direct flow measurements is therefore compelling. Thermodilution is one method for doing $\mathrm{so}^{8}$; with conventional implementations, cold saline is injected upstream, and flow parameters are measured using downstream temperature measurements. However, thermodilution is often too cumbersome for routine use in clinical environments, and there can be significant intra-patient variability in the measurements ${ }^{9}$.

Novel Biophotonics Techniques and Applications V, edited by Arjen Amelink, Seemantini K. Nadkarni, Proc. of SPIE-OSA Vol. 11075, 1107502 - @ 2019 SPIE-OSA · CCC code: 1605-7422/19/\$21 · doi: 10.1117/12.2527056 
In this study, we present a solid-state optical method for performing intravascular flow measurements. In a technique similar to thermodilution, water is thermally tagged upstream; downstream, temperature changes are measured with low coherence interferometry. This paper provides proof-of-concept data for this paradigm, using water as a blood mimicking fluid.

\section{METHODS}

\subsection{Fibre Optic Intravascular Flow Sensor}

The fibre optic flow sensor developed here comprises a single-mode optical fibre, with a dome of polydimethylsiloxane (PDMS) applied to the distal end. When the dome experiences an external temperature change, its optical path length increases. This change in optical path length can then be related to a change in temperature ${ }^{10}$. Temperature measurements were performed using phase-resolved spectral-domain low coherence interferometry (SD-LCI) ${ }^{11}$, a method which continuously interrogates the changes in the length of the dome with nanometre-scale resolution.

The SD-LCI system, shown in Figure 1, includes a broadband single-mode laser source centred at $1550 \mathrm{~nm}$. A commonpath interferometer was formed from the etalon comprising the distal end of the fibre and the outer surface of the PDMS dome, as previously described by Coote et al. ${ }^{10}$. Fourier transforms were performed on interferograms acquired by the spectrometer, and the complex components corresponding to the length of the etalon were selected. Changes in the optical path length of the dome were linearly related to the change in the unwrapped argument of the complex phase ${ }^{10,12}$. A secondary optical fibre delivered light for pulsed upstream thermal tagging (pulse duration $0.4 \mathrm{~s}$; power: $1 \mathrm{~W}$ ). This thermal tagging light, with a centre wavelength of $1470 \mathrm{~nm}$ chosen to correspond to a local maximum of the optical absorption spectrum of water, was delivered through the sides of a fibre optic diffuser over an approximate length range of $1 \mathrm{~mm}$.

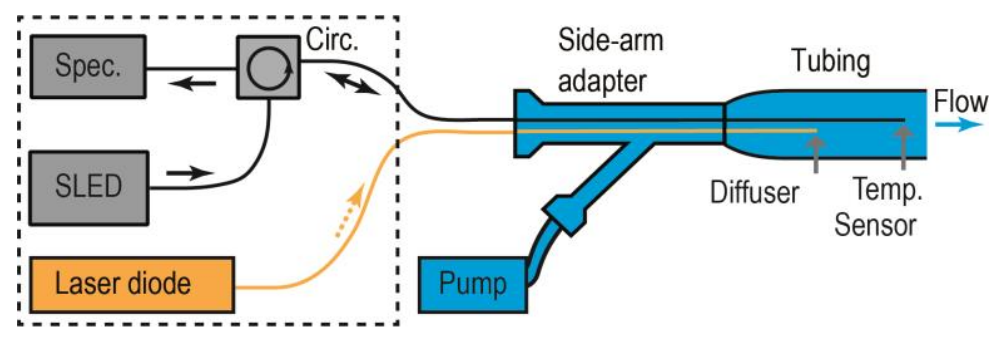

Figure 1. Schematic diagram of the SD-LCI system. A superluminescent light emitting diode (SLED) was used to interrogate the temperature (Temp.) sensor, which comprised a polymer dome at the end of a single-mode fibre; the resulting interference pattern was detected with a spectrometer (Spec.) via a circulator (Circ.). The temperature sensor was positioned into a benchtop flow phantom via a side-arm adaptor, and a syringe pump provided uni-directional flow. An optical diffuser, which provided upstream heating using light from a laser diode $(1470 \mathrm{~nm})$, was positioned upstream from the temperature sensor.

\subsection{Calibration}

Temperature sensor calibration was performed with a temperature-controlled water bath. The water was continuously stirred and its temperature was varied in five steps between 25 to $50{ }^{\circ} \mathrm{C}$. A thermocouple was used to provide a corresponding temperature measurement. The relationship between phase $(\varphi)$, the measurand, and temperature, T, was linear; $\mathrm{dT} / \mathrm{d} \varphi$ was found to be $9.40 \pm 0.19^{\circ} \mathrm{C} / \mathrm{rad}$, for a dome of length $50.4 \mu \mathrm{m}$ (Figure 2). The linear intercept of the calibration was dependent on the temperature of the dome when the phase was first recorded.

\subsection{Benchtop Model}

The benchtop flow phantom consisted of a syringe pump (AL-1000, World Precision Instruments) that injected water through straight plastic tubing and collected by a second syringe. The two optical fibres were positioned into the phantom via a side-arm connector (Figure 1). During pump operation, the diffuser and the temperature sensor were observed to be close to the centre of the vessel, although detailed position estimates were not available. A section of tubing could be swapped to allow for different lumen diameters $(2.5$ to $5 \mathrm{~mm})$. Constant flow rates, which were chosen in the range of 5 to $200 \mathrm{ml} / \mathrm{min}$, were used. These rates were consistent with blood flow in healthy vessels, with diameters similar to those in this phantom. 


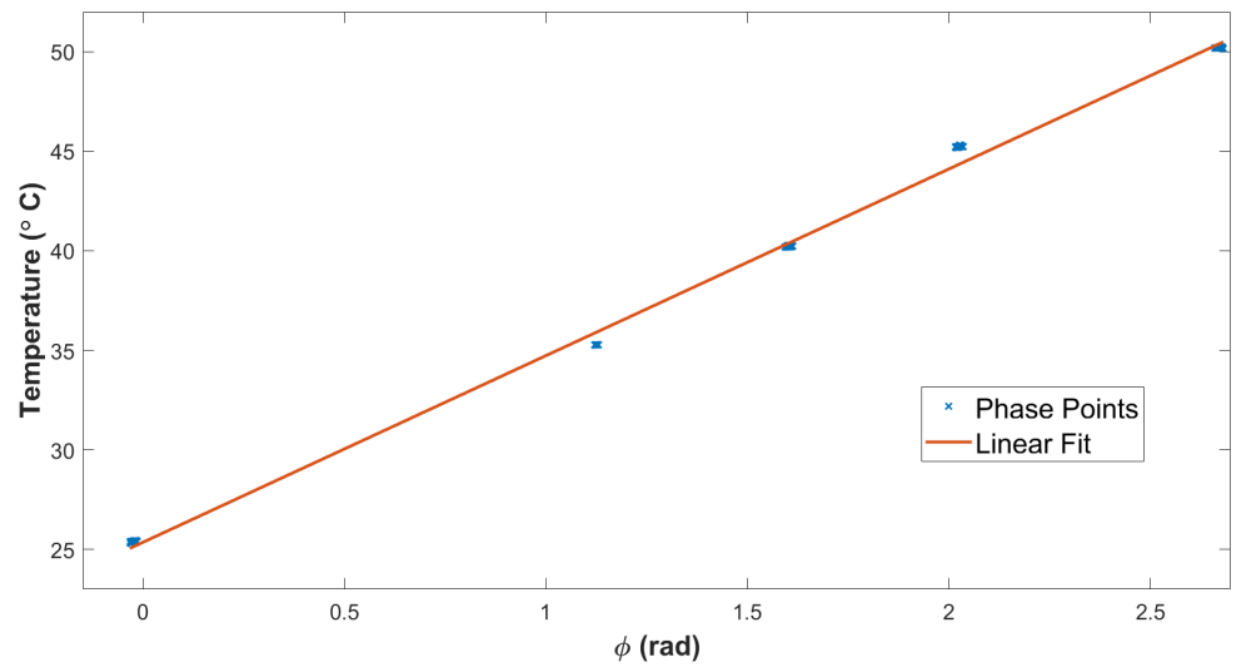

Figure 2. For calibration, the temperature (T) is plotted as a function of the unwrapped complex phase ( $\varphi$ ) of a component of the Fourier transformed interferogram that corresponded to the length of the temperature-sensitive polymer dome. For this flow sensor with a dome length of $50.4 \mu \mathrm{m}$ at room temperature, a linear fit yielded $\mathrm{dT} / \mathrm{d} \varphi=9.40 \pm 0.19^{\circ} \mathrm{C} / \mathrm{rad}$.

\section{RESULTS AND DISCUSSION}

Optical thermal tagging induced measurable increases in downstream temperature. The temperature profiles varied with flow (Figure 3). In particular, the maximum temperature change that was reached, $\mathrm{T}_{\max }$, increased with flow: at $20 \mathrm{ml} / \mathrm{min}$, $\mathrm{T}_{\max }$ was $0.22{ }^{\circ} \mathrm{C}$; at $5 \mathrm{ml} / \mathrm{min}, \mathrm{T}_{\max }$ was $0.08{ }^{\circ} \mathrm{C}$. The temperature profiles also varied in terms of the maximum rate at which temperature increased, $T_{\text {grad: }}$ higher flow resulted in higher maximum rates of temperature increase (data not shown).

A temporal parameter, $t_{\text {base }}$, was calculated by first finding the tangent to the temperature profile at its maximum gradient, and then finding the intercept of that tangent with the baseline temperature. This parameter, which was intended to provide an indication of the minimum detectable transit time for the thermally tagged fluid, decreased with the flow rate for a given vessel diameter (Figure 3). Temperature profiles were also clearly resolved at higher flow rates of 80 to $200 \mathrm{ml} / \mathrm{min}$ (Figure 4).

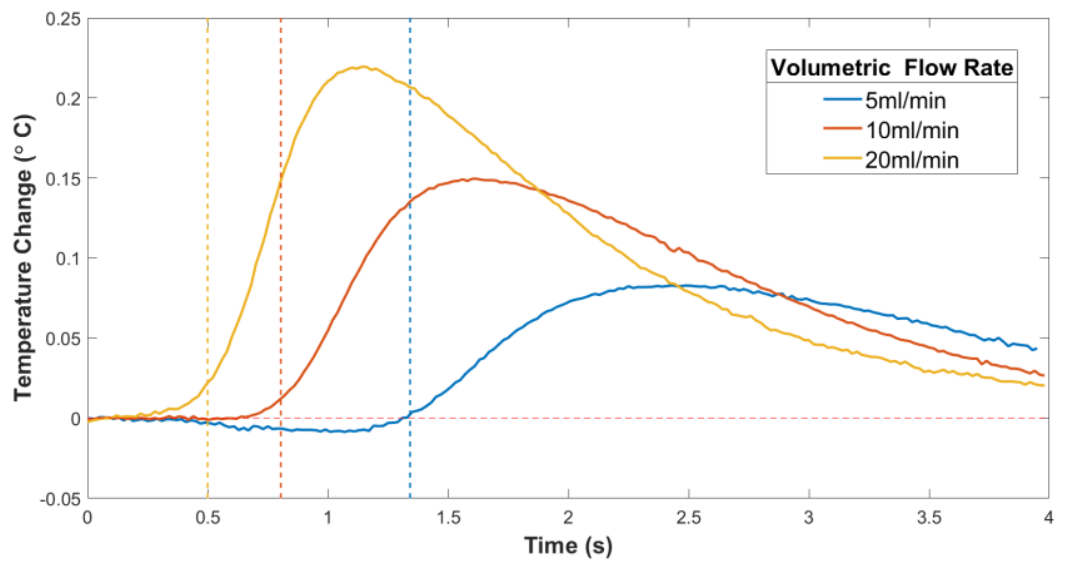

Figure 3. Downstream temperature profiles, expressed as a difference from the baseline temperature (dashed horizontal line) varied with the volumetric flow rate. The vessel diameter was $3 \mathrm{~mm}$. As the flow rate increased, $\mathrm{T}_{\max }$ increased and tbase decreased. The dashed vertical lines correspond to tbase for each flow rate; at $5 \mathrm{ml} / \mathrm{min}$, tbase was $1.34 \mathrm{~s}$, at $10 \mathrm{ml} / \mathrm{min}$, tbase was $0.80 \mathrm{~s}$, and at $20 \mathrm{ml} / \mathrm{min}$, tbase was $0.50 \mathrm{~s}$. 


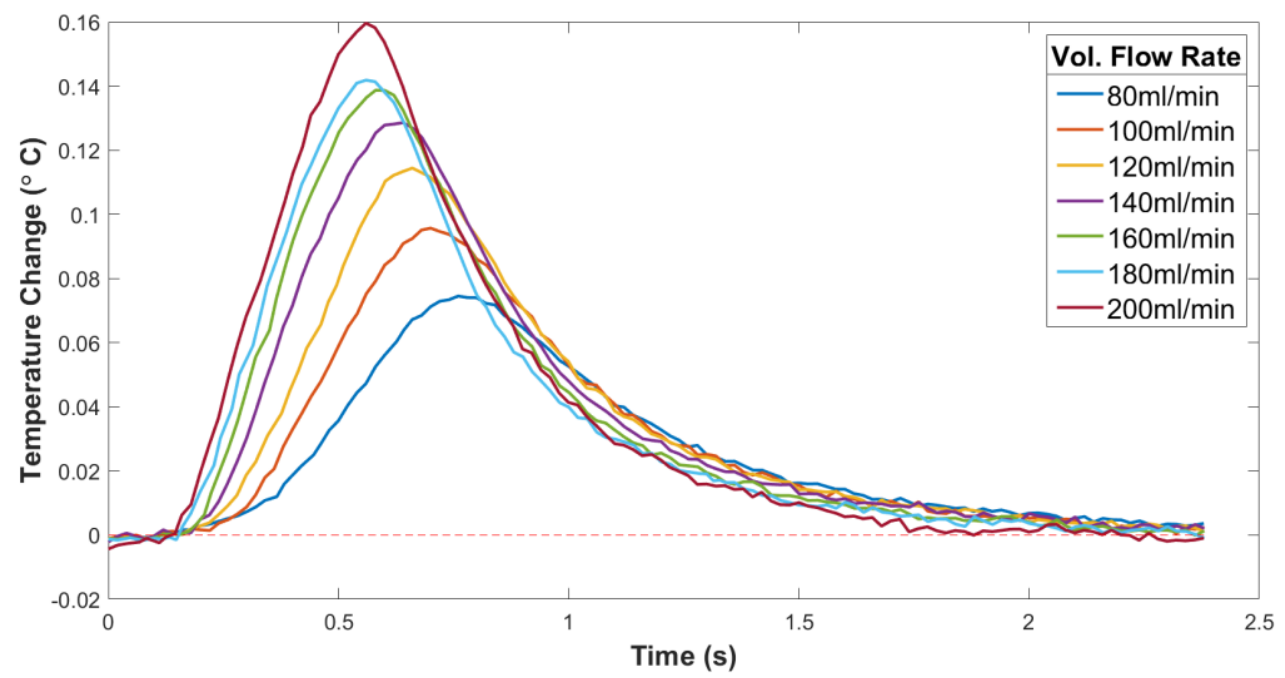

Figure 4. Changes in downstream temperature, expressed as a difference from the baseline temperature (dashed line), after upstream thermal tagging for volumetric flows of 80 to $200 \mathrm{ml} / \mathrm{min}$ (vessel diameter: $2.5 \mathrm{~mm}$ ).

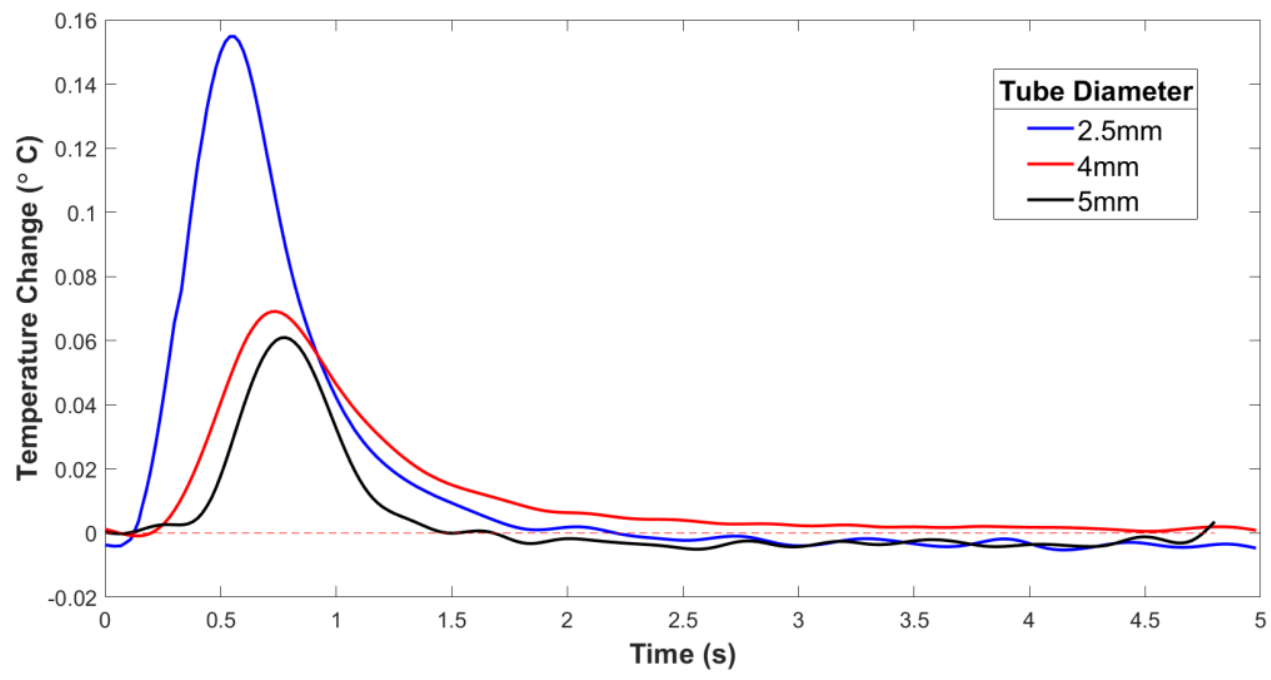

Figure 5. Changes in downstream temperature, expressed as a difference from the baseline temperature (dashed line), after upstream heating for a volumetric flow of $200 \mathrm{ml} / \mathrm{min}$, for different vessel diameters: $2.5,4$, and $5 \mathrm{~mm}$.

Different vessel diameters resulted in different temperature profiles, for a given flow rate (Figure 5). In particular, a larger diameter resulted in a smaller $\mathrm{T}_{\max }$. To further highlight the effect of vessel diameter on the temperature response, Figure 6(a) shows t $t_{\text {base }}$ for three different vessel diameters $(2.5,4$, and $5 \mathrm{~mm}$ ), plotted against a range of volumetric flow rates from 80 to $200 \mathrm{ml} / \mathrm{min}$. Both a smaller diameter vessel and a higher volumetric flow rate resulted in smaller tbase values. These results are consistent with an assumption of laminar flow: in a small diameter vessel, the maximum flow velocity is higher than it would be in a larger diameter vessel, given the same volumetric flow rate, and therefore there is a shorter transit time. 
(a)

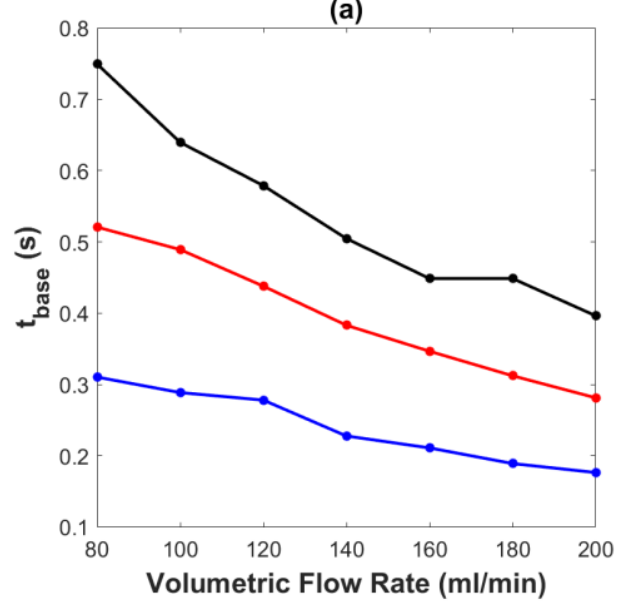

(b)

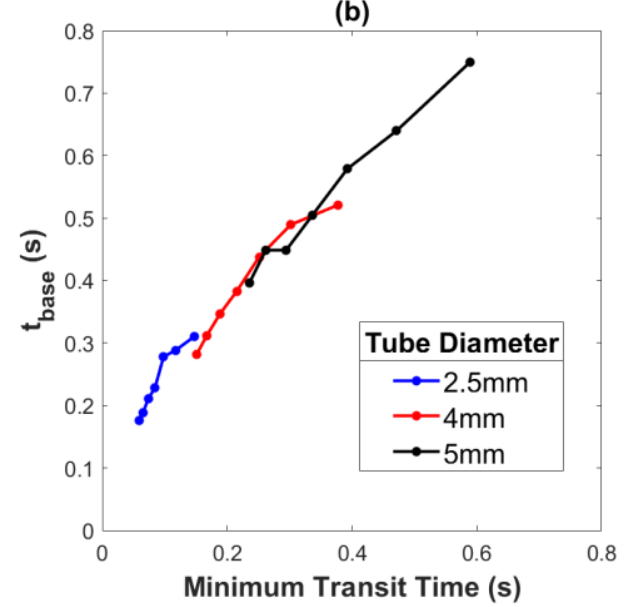

Figure 6: (a) The temporal parameter $t_{\text {base}}$, which was intended to provide an indication of the minimum time required for upstream heating to generate a downstream temperature response, is plotted against the volumetric flow rate for three different vessel diameters; $2.5,4$, and $5 \mathrm{~mm}$. (b) tbase is plotted against the theoretical minimum time of flight, which was calculated as the distance between the heating element and the flow sensor divided by the maximum flow velocity (with the assumption of laminar flow).

The parameter $t_{\text {base }}$ increased with the theoretical minimum transit time for fluid to travel from the diffuser to the temperature sensor, in a manner that was consistent across all three vessel diameters (Figure 6(b)). This minimum transit time was calculated by dividing the distance between the distal end of the fibre optic diffuser and the temperature sensing dome $(2 \mathrm{~cm})$ by the maximum velocity expected for the vessel, given the cross-sectional area and volumetric flow rate, and the assumption of laminar flow. The maximum velocity, $\mathrm{v}_{\max }$, was calculated from:

$$
\mathrm{v}_{\max }=2 \mathrm{Q} / \mathrm{A}
$$

where $\mathrm{Q}$ is the volumetric flow rate and $\mathrm{A}$ is the cross-sectional area.

The temperature profiles resulting from upstream thermal tagging can be expected to depend on several factors, including thermal diffusion in directions parallel and perpendicular to the vessel axis, the locations of the diffuser and the temperature sensor within the vessel, and the presence of components used to position them within the vessel. Simulations and analytic approximations will be valuable to gain a more detailed understanding of these factors. Future experiments will include a larger range of tubing diameters, more realistic blood mimicking fluids, integration into a medical device, and in vivo measurements.

\section{CONCLUSION}

This study provided a proof-of-concept experimental demonstration that physiological flow rates can be measured using a solid-state optical version of thermodilution with upstream thermal tagging. In contrast to thermodilution, fluid injections were not required to effect upstream temperature changes. An important future direction is the measurement of variable flow, as experienced in real coronary arteries. Optical thermal tagging measurements of flow are very promising for use in interventional cardiology; they could potentially provide information that is valuable for decisionmaking on patient treatments that is complementary to existing pressure-based approaches. 


\section{REFERENCES}

[1] P. W. F. Wilson, P. S. Douglas. Epidemiology of coronary heart disease. Technical report, 2018.

[2] American Heart Association Statistics Committee and Stroke Statistics Subcommittee. Heart Disease and Stroke Statistic 2017 Update: A Report From the American Heart Association. Circulation, 135(10):146-603, 2017.

[3] M. J. Davies, N. Woolf. Atherosclerosis: what is it and why does it occur? British Heart Journal, 69(S):3-11, 1993.

[4] ACC/AHA. Guidelines for Percutaneous Transluminal Coronary Angioplasty. Journal of the American College of Cardiology, 12(2):529-45, 1988.

[5] M. J. Kern. Clinical use of coronary artery pressure flow measurements. Technical report, 2018.

[6] A. Baranauskas, A. Peace, A. Kibarskis, J. Shannon, V. Abraitis, V. Bajoras, V. Bilkis, A. Aidietis, A. Laucevicius, G. Davidavicius, and et al. FFR result post PCI is suboptimal in long diffuse coronary artery disease. EuroIntervention, 12(12):1473-1480, 2016.

[7] S. Poeggel, D. Tosi, D. Duraibabu, G. Leen, D. McGrath, E. Lewis. Optical Fibre Pressure Sensors in Medical Applications. Sensors, 15(7):17115-17148, 2015.

[8] W. W. Nichols, M. F. O'Rourke, C. Vlachopoulos, D. A. McDonald. McDonald's Blood flow in arteries: theoretical, experimental and clinical principles. Hodder Arnold, 2011.

[9] J. H. Stevens. Thermodilution cardiac output measurement. Journal of the American Medical Association, 253(15):22402242, 1985.

[10] J. M. Coote, E. J. Alles, S. Noimark, C. A. Mosse, C. D. Little, C. D. Loder, A. L. David, R. D. Rakhit, M. C. Finlay, A. E. Desjardins. Dynamic physiological temperature and pressure sensing with phase-resolved low-coherence interferometry. Optical Express 27(4):5641-5654, 2019.

[11] C. Joo, T. Akkin, B. Cense, B. H. Park, J. F. Boer. Spectral-domain optical coherence phase microscopy for quantitative phase-contrast imaging. Optics Letters, 30(16):2131-2133, 2005.

[12] M. A. Choma, A. K. Ellerbee, C. Yang, T. L. Creazzo, J. A. Izatt. Spectral-domain phase microscopy. Optics Letters, 30(10):1162, 2005. 